\title{
Cystoid macular oedema after extracapsular cataract extraction and intraocular lens implantation in diabetic patients without retinopathy
}

\author{
Ugo Menchini, Francesco Bandello, Rosario Brancato, Fabrizio I Camesasca, Marcello Galdini
}

\begin{abstract}
Postoperative onset or aggravation of cystoid macular oedema (CMO) in diabetic patients after extracapsular cataract extraction (ECCE) with intraocular lens (IOL) implantation is a frequent problem. At present little is known about the occurrence and prognosis of this complication in diabetics with no clinically detectable diabetic retinopathy (DR). Twenty five diabetic eyes ( 24 subjects) without DR and 45 normal eyes (44 subjects) were studied before surgery and $30,90,180,360$ days after ECCE and posterior chamber IOL implantation. Fluorescein angiography was performed at each examination. The frequency of angiographic CMO in the two groups was comparable 30 days after surgery but was significantly higher in diabetic eyes at 90,180 , and 360 days. This finding is probably related to an impairment of the blood-retinal barrier in diabetics. Final visual acuity, however, was similar in the two groups.
\end{abstract}

(Br F Ophthalmol 1993; 77: 208-211)

Cataract extraction in diabetic patients may lead to several complications, particularly during the postoperative period. Removal of the lens eliminates an important barrier and, when ischaemic diabetic retinopathy is present, can lead to the onset or aggravation of iris neovascularisation and eventually to neovascular glaucoma. ${ }^{12}$ The risk is greater with intracapsular than with extracapsular cataract extraction (ECCE).

Several problems related to the evolution of diabetic retinopathy remain poorly understood. One of these is the postoperative onset of cystoid macular oedema (CMO) or the aggravation of existing proliferative diabetic retinopathy. More specifically, there is surprisingly little knowledge on the complications that follow cataract surgery in diabetic patients without clinical signs of retinopathy.

In this study we evaluated the frequency and duration of angiographic CMO after ECCE and intraocular lens (IOL) implantation in the ciliary sulcus in a group of diabetic patients without clinical signs of retinopathy, and in a control group of normal patients.

\section{Patients and methods}

The diabetic patients included in this study underwent cataract extraction at our department between January and July 1990. The normal patients who formed the control group underwent ECCE surgery between January and March
1990. All the patients were operated on by the same surgeon using the same technique.

Twenty five eyes of 24 diabetic patients were included in the first group. Diabetes was defined as a fasting plasma glucose level of $140 \mathrm{mg} / \mathrm{dl}$ or more on at least two occasions. All patients had type II diabetes. These patients showed no preoperative diabetic retinopathy in either eye on clinical and fluorescein angiographic examination; ocular axial length was less than $24.5 \mathrm{~mm}$ in all of them, and none had preoperative ocular or systemic disease.

Similarly, the patients in the control group also had to have an ocular axial length less than $24.5 \mathrm{~mm}$ and no preoperative ocular or systemic disease. This group consisted of 45 eyes in 44 patients. The demographic data for the study patients are presented in Table 1. Each patient underwent complete ophthalmic examination and retinal fluorescein angiography before surgery. Eyes with severe lens opacities that hindered observation of the fundus were excluded from the study.

All the eyes underwent planned ECCE by means of the following technique: a corneoscleral incision was made and the anterior chamber was filled with sodium hyaluronate. A large anterior capsulotomy was then performed. The nucleus was expressed and the cortex of the lens was removed carefully by manual irrigation aspiration. The posterior capsule was polished and left intact in all cases. A posterior chamber c-loop polymethylmethacrylate IOL was positioned in the ciliary sulcus. No adrenaline was added to the infusion used for irrigation, and acetylcholine was not added after IOL implantation. The corneoscleral cut was closed with a continuous 10-0 nylon suture. A subconjunctival injection of $250 \mathrm{mg}$ of piperacillin and $1 \mathrm{mg}$ of betamethasone was given at the end of surgery.

During the immediate postoperative period, topical therapy included fluocinolone and neo-

Table 1 Demographic data for 24 diabetic and 44 normal patients

\begin{tabular}{lll}
\hline & Diabetic & Normal \\
\hline No of patients & 24 & 44 \\
No of eyes & 25 & 45 \\
Males & $11(45 \cdot 8 \%)$ & $19(43 \cdot 2 \%)$ \\
Females & $13(54 \cdot 2 \%)$ & $25(56 \cdot 8 \%)$ \\
Age at cataract extraction (years) & $69 \cdot 8(10 \cdot 7)$ & $70 \cdot 3(10 \cdot 1)$ \\
Mean (SD) & 48 to 89 & 51 to 88 \\
Range & $7 \cdot 6(7 \cdot 1)$ & \\
Duration of diabetes (years) & 1 to 20 & \\
Mean (SD) & $5(20 \cdot 8 \%)$ & \\
Range & $16(66 \cdot 7 \%)$ & \\
Treatment of diabetes & $3(12 \cdot 5 \%)$ & \\
Insulin & \\
Oral hypoglycaemic agents & \\
Dietary restriction & \\
\hline
\end{tabular}


Table 2 Frequency of fluorescein angiographic CMO 30, 90,180 , and 360 days after extracapsular cataract extraction in diabetic eyes without retinopathy and normal eyes.

Statistical analysis of CMO frequency in both groups is reported (Fisher's exact test)

\begin{tabular}{|c|c|c|c|}
\hline Days & & Diabetic & Normal \\
\hline 30 & $\begin{array}{l}\text { CMO } \\
\text { no CMO }\end{array}$ & $\begin{array}{l}(n=16) \\
68 \cdot 8 \%(n=11) \\
31 \cdot 2 \%(n=5)\end{array}$ & $\begin{array}{l}(n=40) \\
62 \cdot 5 \%(n=25) \\
37 \cdot 5 \%(n=15)\end{array}$ \\
\hline 90 & $\begin{array}{l}\text { CMO } \\
\text { no CMO }\end{array}$ & $\begin{array}{l}(n=20) \quad p=0.452 \\
65 \cdot 0 \%(n=13) \\
35 \cdot 0 \%(n=7)\end{array}$ & $\begin{array}{l}(\mathrm{n}=45) \\
33 \cdot 3 \%(\mathrm{n}=15) \\
66 \cdot 7 \%(\mathrm{n}=30)\end{array}$ \\
\hline 180 & $\begin{array}{l}\text { CMO } \\
\text { no CMO }\end{array}$ & $\begin{array}{l}(n=14) \quad p=0 \cdot 017 \\
42 \cdot 9 \%(n=6) \\
57 \cdot 1 \%(n=8)\end{array}$ & $\begin{array}{c}(n=36) \\
8 \cdot 3 \%(n=3) \\
91 \cdot 7 \%(n=33)\end{array}$ \\
\hline 360 & $\begin{array}{l}\text { CMO } \\
\text { no CMO }\end{array}$ & $\begin{array}{l}(n=25) \quad p=0.009 \\
24.0 \%(n=6) \\
76.0 \%(n=19) \\
\quad p=0.0013\end{array}$ & $\begin{array}{c}(n=45) \\
0 \cdot 0 \%(n=0) \\
100 \cdot 0 \%(n=45)\end{array}$ \\
\hline
\end{tabular}

mycin eye drops four times daily plus tropicamide eye drops once a day. In no case were eye drops containing adrenaline derivatives instilled. The patients were examined thoroughly 30,90 , 180 , and 360 days after surgery, and retinal fluorescein angiography was performed at all these intervals. Informed consent was obtained in all cases.

At ophthalmoscopical examination, CMO appears to be composed of cystic spaces located in the macular region and filled with clear fluid. Retinal fluorescein angiography highlights the typical petaloid distribution of these cystic spaces. In this study, the minimal change that was considered positive for angiographic CMO was the presence of fluorescein pooling in the macular region with less than perifoveal distribution (incomplete petaloid changes) on late phase angiograms. ${ }^{3}$ Early and late phase angiograms were evaluated independently by two of the authors.

The difference in the frequency of angiographic CMO between the eyes of normal and diabetic patients at each follow up was analysed by Fisher's exact test. Furthermore, the correlation between the duration of diabetes and the presence of angiographic $\mathrm{CMO}$ at each follow up was analysed by Fisher's exact test. Ten year duration of diabetic disease was chosen as cut off point.

\section{Results}

No patient in either group showed any ocular or systemic disease that could have affected the postoperative course (that is, high myopia, severe pigment changes in the macula, glaucoma, systemic hypertension), and in no case was the posterior capsule broken during surgery. $\mathrm{Nd}$ :

Table $3 a$ Corrected visual acuity-normal eyes

\begin{tabular}{lccll}
\hline \multicolumn{5}{c}{ Postoperative days } \\
\cline { 2 - 5 } Visual & $30(n=40)$ & $90(n=45)$ & $180(n=36)$ & $360(n=45)$ \\
acuity & $N o(\%)$ & $N o(\%)$ & $N o(\%)$ & $N o(\%)$ \\
\hline $6 / 6->6 / 7 \cdot 5$ & $6(15 \cdot 0)$ & $22(48 \cdot 9)$ & $20(55 \cdot 6)$ & $29(64 \cdot 5)$ \\
$6 / 7 \cdot 5-6 / 12$ & $28(70 \cdot 0)$ & $20(44 \cdot 4)$ & $13(36 \cdot 1)$ & $14(31 \cdot 1)$ \\
$6 / 15-6 / 30$ & $6(15 \cdot 0)$ & $3(6 \cdot 7)$ & $3(8 \cdot 3)$ & $2(4 \cdot 4)$ \\
\hline
\end{tabular}

*Visual acuity is expressed in $6 \mathrm{~m}$ standard.
Table $3 b$ Corrected visual acuity - diabetic eyes

\begin{tabular}{lllll}
\hline \multicolumn{5}{c}{ Postoperative days } \\
\cline { 2 - 5 } Visual & $30(n=16)$ & $90(n=20)$ & $180(n=14)$ & $360(n=25)$ \\
acuity & $N o(\%)$ & $N o(\%)$ & $N o(\%)$ & $N o(\%)$ \\
\hline $6 / 6->6 / 7 \cdot 5$ & $3(18 \cdot 7)$ & $6(30 \cdot 0)$ & $6(42 \cdot 9)$ & $13(52 \cdot 0)$ \\
$6 / 7 \cdot 5-6 / 12$ & $8(50 \cdot 0)$ & $8(40 \cdot 0)$ & $5(35 \cdot 7)$ & $11(44 \cdot 0)$ \\
$6 / 15-6 / 30$ & $5(31 \cdot 3)$ & $6(30 \cdot 0)$ & $3(21 \cdot 4)$ & $1(4 \cdot 0)$ \\
\hline
\end{tabular}

^Visual acuity is expressed in $6 \mathrm{~m}$ standard.

YAG laser capsulotomy was not required in any case.

Results and statistical analysis are reported in Table 2. The two groups appeared homogeneous when compared for age and sex. Some patients either did not show up for the intermediate follow ups, or refused fluorescein angiography, but all were examined 360 days after surgery. The frequency of angiographic CMO in normal and diabetic eyes was the same $\mathbf{3 0}$ days after surgery, but there was a significant difference at 90 and 180 days. In almost all cases with complete follow up, onset of fluorescein angiographic CMO was observed at 30 days $(21 / 22$ normals, 9/10 diabetics). In two cases only CMO was initially detected at 90 days. One year after surgery, $24.0 \%$ of the diabetic eyes still had angiographic CMO, while none of the normal eyes was affected. In no case did a diabetic eye develop angiographically or clinically detectable diabetic retinopathy. Interobserver variability was low (5\%).

There was no correlation between the presence of angiographic CMO and the duration of diabetes at every follow up. This was valid for diabetic disease, respectively, of 10 years or less, and of more than 10 years in duration.

Visual acuity in the normal eyes was better than in the diabetic eyes at every follow up (Tables 3a and $3 b$ ), but this difference was not significant.

Table 4 reports mean visual acuity in the two groups at the four follow up intervals. In order to assess the influence of CMO and diabetes on visual acuity, we performed a two way analysis of variance (general linear models procedure). The results indicated that 30 days after surgery neither CMO nor diabetes were connected with visual acuity, while at the 90 day examination the presence of diabetes was related $(p=0.03)$ with a worst visual acuity. Finally, 180 and 360 days after surgery, the presence of CMO was, respectively, close to significance $(\mathrm{p}=0.06)$ and significantly related $(p=0.03)$ with a lower visual acuity.

Table 4 Average visual acuity of normal and diabetic eyes without retinopathy, with and without angiographic cystoid macular oedema (CMO), at the follow ups. (Visual acuity is expressed in $6 m$ standard.)

\begin{tabular}{llll}
\hline Days & & $\begin{array}{l}\text { Diabetic } \\
\text { Mean }(S D)\end{array}$ & $\begin{array}{l}\text { Normal } \\
\text { Mean }(S D)\end{array}$ \\
\hline \multirow{2}{*}{30} & CMO & $(\mathrm{n}=16)$ & $(\mathrm{n}=40)$ \\
& no CMO & $13 \cdot 9(7 \cdot 6)$ & $10 \cdot 1(4 \cdot 6)$ \\
90 & $9 \cdot 6(2 \cdot 3)$ & $12 \cdot 6(7 \cdot 9)$ \\
$(\mathrm{n}=45)$ \\
90 & CMO & $11 \cdot 9(7 \cdot 2)$ & $8 \cdot 8(3 \cdot 0)$ \\
& no CMO & $10 \cdot 3(2 \cdot 8)$ & $8 \cdot 0(3 \cdot 3)$ \\
180 & CMO & $(\mathrm{n}=14)$ & $(\mathrm{n}=36)$ \\
& no CMO & $13 \cdot 5(9 \cdot 0)$ & $9 \cdot 0(3 \cdot 0)$ \\
360 & CMO & $7 \cdot 7(2 \cdot 2)$ & $8 \cdot 1(3 \cdot 5)$ \\
& no CMO & $9 \cdot 8(3 \cdot 8)$ & $(\mathrm{n}=45)$ \\
& & $7 \cdot 0(1 \cdot 7)$ & $7 \cdot 7(3.9)$ \\
\hline
\end{tabular}




\section{Discussion}

Clinical CMO has been defined as reduction in prior visual acuity associated with ophthalmoscopically visible cystoid changes in the macula or angiographic evidence of $\mathrm{CMO} .{ }^{4}$ Angiographic CMO has been described as CMO detected with retinal fluorescein angiography in patients who do not have noticeable disturbance in their vision. ${ }^{3}$

The incidence of CMO after ECCE in normal subjects is higher 4-12 weeks after surgery, although CMO may occur months or even years later. ${ }^{5}$ Angiographic CMO has been reported in $10 \%$ of those cases with an intact posterior capsule. ${ }^{5}$ This anomalous permeability of the macular capillary network, however, along with that of the optic nerve head, usually improves after about 3 months. Clinical CMO after ECCE and posterior chamber IOL implantation becomes chronic in almost $1 \%$ of cases, ${ }^{46-8}$ causing a progressive impairment of vision. Alpar reported a $13.3 \%$ and $11 \cdot 1 \%$ incidence of, respectively, clinical and angiographic CMO in diabetic patients 2 years after cataract surgery and IOL implantation in the sulcus.'

The known causes of post-cataract CMO are: mechanical (vitreous or iris incarceration in the surgical wound), iatrogenic (instillation of adrenaline and its derivatives), inflammatory (continuous iris irritation by an iris-fixated IOL), and physical (ultraviolet radiation reaching the retina when the lens is removed). The presence and integrity of the posterior lens capsule is an important element. ${ }^{610}$

A breakdown of the blood iris barrier in aphakic patients indicates that the inflammatory agent causing $\mathrm{CMO}$ affects the capillary network in several ocular districts. ${ }^{11}$

Improvements in surgical technique, such as the development of ECCE, have significantly reduced the frequency of CMO. ${ }^{46-8}$ This technique preserves the compartmentalisation of the anterior and posterior segments of the eye, and the inflammatory agents released in the anterior chamber during surgery do not easily reach the retina. Furthermore, the posterior lens capsule prevents anterior vitreous displacement. We think that there is a threshold above which inflammatory agents lead to breakdown of the inner retinal barrier with consequent CMO. The action of these agents is greater in the weeks immediately after surgery and progressively decreases over a period of a few weeks.

The first sign of diabetes induced changes in the retina is the breakdown of the blood-retinal barrier. ${ }^{12}$ Diabetes induces changes of the inner retinal barrier that are histologically evidenced by hypertrophy of the endothelial cells, increased basement membrane thickness, and loss of pericytes. ${ }^{13}$ Loss of pericytes occurs early in the disease, and leads to an increase in vascular diameter and permeability. ${ }^{12}{ }^{14}$ The outer retinal barrier is affected as well, with changes and disorganisation of the retinal pigment epithelium cells. ${ }^{13}$ is The fluid transport capacity of the retinal pigment epithelium appears compromised in diabetes and this could be a cause of macular oedema. ${ }^{13}$

When inflammation consequent to cataract surgery affects the diabetic patient's vascular walls, it may lead to an increase in permeability more easily and with greater severity than in the non-diabetic subject, with a higher incidence of CMO and iris-blood barrier breakdown in consequence. ${ }^{11}$

In our study, this increase in permeability and the consequent angiographic CMO was comparable in normal and diabetic patients without retinopathy at 30 days after surgery. However, a significant difference was observed at the 90, 180 , and 360 day follow ups. We believe that even a moderate inflammatory stimulus that persists can in time alter vascular permeability in diabetic patients. It is possible, however, simply that it is more difficult to restore a normal barrier function in diabetic eyes, even when diabetic retinopathy is absent.

No significant correlation was found in this study between duration of diabetes and the presence of CMO. A possible explanation for this observation may be the fact that the time of diagnosis of type II diabetes unfortunately does not always reflect the real duration of the disease, which may remain undetected for several years.

It is worth noting that, despite the difference in the fluorescein angiographic CMO rate, there was no significant difference in visual function between normal controls and diabetic patients without retinopathy. This is consistent with previous studies. ${ }^{1016}$

The diagnosis of angiographic CMO in this study was made by trained observers who examined early and late phase fluorescein angiograms. Inter and intraobserver variations are among the known drawbacks of this subjective method. Recently developed computerised quantitative image analysis appears to be a promising and effective method providing more accurate, objective quantitative evaluation of macular leakage. ${ }^{17}$

This work was presented at the lst Meeting of the European Association for the Study of Diabetic Eye Complication (EASDEC) London, UK, 8-9 September 1991.

1 Poliner LS, Christianson DJ, Escoffery RF, Kolker AE Gordon ME. Neovascular glaucoma after intracapsular and extracapsular cataract extraction in diabetic patients Am f Ophthalmol 1985; 100: 637-43.

2 Rice TA, Michels RG, Maguire MG, Rice EF. The effect of lensectomy on the incidence of iris neovascularization and neovascular glaucoma after vitrectomy for diabetic retinopathy. Am F Ophthalmol 1983; 95: 1-11.

3 Yannuzzi LA. A perspective on the treatment of aphakic cystoid macular edema. Surv Ophthalmol 1984; 28: 540-52.

4 Stark WJ Jr, Maumenee AE, Fagadau W, Datiles M, Baker CC, Worthen D, et al. Cystoid macular edema in pseudoCC, Worthen D, et al. Cystoid macular edema in

5 Goodman DF, Stark WJ, Gotsch JD. Complications of Goodman DF, Stark WJ, Gottsch JD. Complications of cataract extraction with intraocu
Ophthalmic Surg 1989; 20: 132-40.

6 Severin TD, Severin SL. Pseudophakic cystoid macular edema: a revised comparison of the incidence with intracapsular and extracapsular cataract extraction. Ophthalmic Surg 1988; 19: 116-8.

7 Jaffe NS, Clayman HM, Jaffe MS. Cystoid macular edema after intracapsular and extracapsular cataract extraction with and without an intraocular lens. Ophthalmology 1982; 89: 25-9:

8 Wetzig PC, Thatcher DB, Christiansen JM. The intracapsular versus the extracapsular cataract technique in relationship to retinal problems. Trans Am Ophthalmol Soc 1979; 77: retinal $339-47$.

9 Alpar JJ. Diabetes: cataract extraction and intraocular lens. $\mathcal{f}$ Cataract Refract Surg 1987; 13: 43-6.

10 Cunliffe IA, Flanagan DW, George NDL, Aggarwaal RJ Moore AT. Extracapsular cataract surgery with lens Moore AT. Extracapsular cataract surgery with lens implantation in diabetics with and without

11 Brancato R, Bandello F, Carnevalini A, Camesasca F, Meloni $P$. Intraocular lens and diabetes. In: Maumenee AE, Stark WJ, Esente I, eds. Proceedings IV International Congress on 
Cataract and Refractive Microsurgery. Milan: Fogliazza, 1990: 133-9.

12 Cunha-Vaz JG. Studies on the pathophysiology of diabetic retinopathy. The blood-retinal barrier in diabetes. Diabetes 1983; 32 (suppl 2): 20-7.

13 Marano CW, Matschinsky FM. Biochemical manifestations of diabetes mellitus in microscopic layers of cornea and retina. Diabetes Metab Rev 1989; 5: 1-15.

14 Lee TS, Hu KQ, Chao T, King GL. Characterization of endothelin receptors and effects of endothelin on diacyl- glycerol and protein kinase $\mathrm{C}$ in retinal capillary pericytes. Diabetes 1989; 38: $1643-6$.

15 Porta M, La Selva M, Molinatti P, Molinatti GM. Endothelial cell function in diabetic microangiopathy. Diabetologia

16 Cheng H, Franklin SL. Treatment of cataract in diabetics with and without retinopathy. Eye 1988; 2: 607-14

17 Phillips RP, Spencer T, Ross PGB, Sharp PF, Forrester JV. Quantification of diabetic maculopathy by digital imaging of the fundus. Eye 1991; 5: 130-7. 